\title{
Frequency and predictors of complications in neurological surgery: national trends from 2006 to 2011
}

\author{
Clinical article
}

\author{
John D. Rolston, M.D., Ph.D., Seunggu J. Han, M.D., Catherine Y. Lau, M.D., \\ Mitchel S. Berger, M.D., and Andrew T. Parsa, M.D., Ph.D. \\ Department of Neurological Surgery, University of California, San Francisco, California
}

\begin{abstract}
Object. Surgical complications increase the cost of health care worldwide and directly contribute to patient morbidity and mortality. In an effort to mitigate morbidity and incentivize best practices, stakeholders such as health insurers and the US government are linking reimbursement to patient outcomes. In this study the authors analyzed a national database to determine basic metrics of how comorbidities specifically affect the subspecialty of neurosurgery.

Methods. Data on 1,777,035 patients for the years 2006-2011 were acquired from the American College of Surgeons (ACS) National Surgical Quality Improvement Program (NSQIP) database. Neurosurgical cases were extracted by querying the data for which the surgical specialty was listed as "neurological surgery." Univariate statistics were calculated using the chi-square test, and 95\% confidence intervals were determined for the resultant risk ratios. A multivariate model was constructed using significant variables from the univariate analysis $(\mathrm{p}<0.05)$ with binary logistic regression.

Results. Over 38,000 neurosurgical cases were analyzed, with complications occurring in 14.3\%. Cranial cases were 2.6 times more likely to have complications than spine cases, and African Americans and Asians/Pacific Islanders were also at higher risk. The most frequent complications were bleeding requiring transfusion (4.5\% of patients) and reoperation within 30 days of the initial operation (4.3\% of patients), followed by failure to wean from mechanical ventilation postoperatively $(2.5 \%)$. Significant predictors of complications included preoperative stroke, sepsis, blood transfusion, and chronic steroid use.

Conclusions. Understanding the landscape of neurosurgical complications will allow better targeting of the most costly and harmful complications of preventive measures. Data from the ACS NSQIP database provide a starting point for developing paradigms of improved care of neurosurgical patients.

(http://thejns.org/doi/abs/10.3171/2013.10.JNS122419)
\end{abstract}

KeY Words • complication •

quality improvement

- $\quad$ adverse event

error

A $\mathrm{s}$ health care costs mount higher, invested stakeholders are scrutinizing surgical practices with an eye toward improving value-defined as the patient's outcome relative to the cost of care. ${ }^{16}$ Insurers, patients, and government agencies are all beginning to make decisions about health care based on perceived value. Surgical complications are often broadly defined as "any deviation from the normal postoperative course." This definition is clearly subjective and prone to interobserver variability. ${ }^{11}$ Within the past several years, nationwide databases, such as those hosted by the Cleveland Clinic (Cardiovascular Information Registry), ${ }^{11}$ the Agency for Healthcare Research and Quality (Patient Safety Indicators), ${ }^{9,17}$ and the American College of Sur-

\footnotetext{
Abbreviations used in this paper: ACS = American College of Surgeons; CPT = Current Procedural Terminology; NSQIP = National Surgical Quality Improvement Program.
}

geons (ACS) (National Surgical Quality Improvement Program [NSQIP]), have begun prospectively collecting data on surgical complications using objective criteria. ${ }^{8,10}$

In particular, the NSQIP database has strict criteria for defining a specified set of frequent postsurgical complications. Data are derived from hundreds of US hospitals in a wide range of settings, from small rural community hospitals to major academic medical centers. In 2006, there were 121 participating sites, and in 2013, there are more than 400 sites. All major surgical procedures performed are identified using Current Procedural Terminology (CPT) codes, and the standardized set of variables is collected from either all cases for lowvolume hospitals or a sample group representing $20 \%$ of all cases performed for large-volume sites. The NSQIP utilizes trained raters to identify these complications directly from the medical records. The raters are frequently audited using test cases, and their data are ignored if they fail these auditing checks. ${ }^{19}$ 


\section{Complications in neurosurgery}

With the NSQIP data set, it is possible to determine the most frequent complications in neurological surgery. Identifying these complications will allow us to better distribute resources to prevent and mitigate the most costly and common adverse events in our patients. Moreover, understanding the type and frequency of complications in neurosurgery will allow us to better coordinate care with stakeholders-insurers, governmental institutions, and patients. In this study, we used the NSQIP database to examine trends and predictors of complications in neurosurgical patients.

\section{Methods}

Data for the years 2006 to 2011 were obtained from the ACS NSQIP database. Files were acquired in delimited text format and parsed using both SPSS version 20 (IBM Corp.) and Matlab R2012a (MathWorks Inc.). Neurosurgical cases were extracted by querying the data for which the primary surgeon's specialty was listed as "neurological surgery," and then each of the CPT codes was individually reviewed to determine whether the case was cranial or spinal (Table 1).

Descriptive statistics are represented as the means \pm standard deviation. Univariate statistics were calculated using the chi-square test, and 95\% confidence intervals are presented for the resultant risk ratios. A multivariate model was constructed with the significant variables from univariate analysis $(p<0.05)$ using binary logistic regression with a maximum number of iterations of 20 . Significant predictors from this model were identified, and their risk ratios were presented, along with the $95 \%$ confidence intervals. Data were plotted using Matlab.

\section{Results}

Records from 1,777,035 surgical cases were compiled. Neurosurgeons performed 38,396 of these cases. Characteristics of the neurosurgical patients are presented in Table 2 and are compared with those of general surgical patients to reveal the unique features of neurosurgical patients while highlighting the landscape of complications encountered. Of note, compared with nonneurosurgical patients, the neurosurgical patients were more likely to be male and white. African American patients had a RR of 0.75 (95\% CI $0.72-0.78)$ of undergoing neurosurgery as opposed to any other surgery, and Asians/Pacific Islanders had an RR of 0.75 (95\% CI 0.70-0.81).

As might be expected, neurosurgical cases were far more likely to involve inpatient procedures rather than outpatient (outpatient RR 0.31, 95\% CI 0.30-0.32). However, neurosurgery was less likely to be done emergently compared with nonneurosurgical procedures (RR 0.46 , 95\% CI 0.44-0.48). Moreover, neurosurgical procedures were significantly longer than nonneurosurgical ones, both in terms of anesthesia time (76.9 minutes, $\mathrm{p}$ $<0.0001)$ and operative time $(52.6$ minutes, $\mathrm{p}<0.0001)$. Neurosurgical patients also stayed on average 0.5 day longer than the other surgical patients $(\mathrm{p}<0.0001)$.

Other demographic features were also compared between neurosurgical and nonneurosurgical patients. Neu-
TABLE 1: CPT codes included in an analysis of neurosurgical complications

\begin{tabular}{|c|c|}
\hline Spine Codes & Cranial Codes \\
\hline 0090T & 21137 \\
\hline 0092T & 21139 \\
\hline 0093T & 21180 \\
\hline 0095T & 21299 \\
\hline 0096T & $35001-5$ \\
\hline 0163T & 35201 \\
\hline 0195T & 35301 \\
\hline 0196T & 35800 \\
\hline 0202T & 37605 \\
\hline 10121 & 37799 \\
\hline 10140 & 49422 \\
\hline 10180 & 60600 \\
\hline 11000 & $61150-888$ \\
\hline $11042-4$ & $62000-362$ \\
\hline 20005 & 69511 \\
\hline 20100 & \\
\hline 20696 & \\
\hline 20902 & \\
\hline 20922 & \\
\hline 20930 & \\
\hline 20931 & \\
\hline 20936 & \\
\hline 20938 & \\
\hline 20999 & \\
\hline 21026 & \\
\hline 21501 & \\
\hline 21510 & \\
\hline 21610 & \\
\hline 21615 & \\
\hline 22010 & \\
\hline 22015 & \\
\hline $22100-14$ & \\
\hline $22206-26$ & \\
\hline $22318-27$ & \\
\hline 22520-95 & \\
\hline $22600-32$ & \\
\hline $22800-99$ & \\
\hline 27080 & \\
\hline 27202 & \\
\hline 27218 & \\
\hline 27280 & \\
\hline 42725 & \\
\hline 49215 & \\
\hline $63001-308$ & \\
\hline 63685 & \\
\hline $63700-41$ & \\
\hline $64573-85$ & \\
\hline
\end{tabular}


J. D. Rolston et al.

TABLE 2: Summary of characteristics in neurosurgical compared with nonneurosurgical patients

\begin{tabular}{|c|c|c|c|c|}
\hline Characteristic & $\begin{array}{l}\text { Neurosurgical } \\
\text { Patients (\%) }\end{array}$ & $\begin{array}{c}\text { Nonneurosurgical } \\
\text { Patients (\%) }\end{array}$ & p Value & $\operatorname{RR}(95 \% \mathrm{Cl})$ \\
\hline total no. of patients & 38,396 & $1,738,639$ & & \\
\hline mean age $(y r s) \pm S D$ & $56.0 \pm 15.0$ & $55.7 \pm 17.0$ & $<0.0001$ & \\
\hline mean height (in) $\pm S D$ & $66.9 \pm 4.2$ & $66.2 \pm 4.1$ & $<0.0001$ & \\
\hline mean weight $(\mathrm{lb}) \pm \mathrm{SD}$ & $186.7 \pm 46.9$ & $186.2 \pm 54.4$ & $<0.0001$ & \\
\hline mean anesthesia time (mins) $\pm S D$ & $238.4 \pm 129.2$ & $161.5 \pm 106.3$ & $<0.0001$ & \\
\hline mean op time (mins) $\pm S D$ & $162.6 \pm 110.1$ & $110.0 \pm 91.3$ & $<0.0001$ & \\
\hline mean length of stay (days) \pm SD & $4.8 \pm 10.2$ & $4.3 \pm 9.9$ & $<0.0001$ & \\
\hline \multicolumn{5}{|l|}{$\operatorname{sex}^{*}$} \\
\hline male & $19,521(50.8)$ & $737,714(42.4)$ & & $1 \dagger$ \\
\hline female & $18,800(49.0)$ & $999,673(57.5)$ & & $0.72(0.70-0.73) \ddagger$ \\
\hline \multicolumn{5}{|l|}{ race $^{*}$} \\
\hline white & $30,360(79.1)$ & $1,319,127(75.9)$ & & $1 \dagger$ \\
\hline black or African American & $2,943(7.7)$ & $171,462(9.9)$ & & $0.75(0.72-0.78) \ddagger$ \\
\hline Asian/Pacific Islander & $731(1.9)$ & $42,327(2.4)$ & & $0.75(0.70-0.81) \ddagger$ \\
\hline American Indian or native Alaskan & $225(0.6)$ & $11,992(0.7)$ & & $0.82(0.72-0.93) \ddagger$ \\
\hline unknown & $4,137(10.8)$ & $193,731(11.1)$ & & \\
\hline \multicolumn{5}{|l|}{ inpatient vs outpatient* } \\
\hline inpatient & $32,871(85.6)$ & $1,121,524(64.5)$ & & $1 \dagger$ \\
\hline outpatient & $5,525(14.4)$ & $617,112(35.5)$ & & $0.31(0.30-0.32) \ddagger$ \\
\hline \multicolumn{5}{|l|}{ diabetes* } \\
\hline no & $32,918(85.7)$ & $1,477,932(85.0)$ & & $1 \dagger$ \\
\hline yes & $5,478(14.3)$ & $260,704(15.0)$ & & $0.94(0.92-0.97) \ddagger$ \\
\hline insulin-dependent & $1,925(5.0)$ & $102,225(5.9)$ & & $0.85(0.81-0.89) \ddagger$ \\
\hline not insulin-dependent & $3,553(9.3)$ & $158,479(9.1)$ & & $1.00(0.97-1.04)$ \\
\hline \multicolumn{5}{|l|}{ tobacco use* } \\
\hline no & $28,693(74.7)$ & $1,391,997(80.1)$ & & $1 \dagger$ \\
\hline yes & $9,702(25.3)$ & $346,621(19.9)$ & & $1.35(1.32-1.38) \ddagger$ \\
\hline \multicolumn{5}{|c|}{ hypertensive patient on antihypertensive(s) ${ }^{*}$} \\
\hline no & $20,392(53.1)$ & $936,936(53.9)$ & & $1 \dagger$ \\
\hline yes & $18,004(46.9)$ & $801,689(46.1)$ & & $1.03(1.01-1.05) \ddagger$ \\
\hline \multicolumn{5}{|l|}{ emergency case ${ }^{*}$} \\
\hline no & $36,108(94.0)$ & $1,525,718(87.8)$ & & $1 \dagger$ \\
\hline yes & $2,288(6.0)$ & $212,916(12.2)$ & & $0.46(0.44-0.48) \ddagger$ \\
\hline
\end{tabular}

rosurgery patients were less likely to have diabetes (RR $0.94,95 \%$ CI $0.92-0.97$ ) but more likely to be on medication for hypertension (RR 1.03, 95\% CI 1.01-1.05). Neurosurgery patients were also more likely to smoke (RR $1.35,95 \%$ CI $1.32-1.38$ ).

In neurosurgical patients alone, we evaluated the frequency of complications documented in the NSQIP database. Cases were split into spinal $(28,017$ cases $)$ and cranial (10,041 cases) surgery groups, and complications were analyzed independently (Table 3); the other 338 cases represented peripheral nerve and nonneurosurgical cases performed by neurosurgeons. Complications occurred in $14.3 \%$ (5507 of 38,396$)$ of neurosurgical cases.
Cranial procedures were significantly more likely to produce complications than spinal procedures (RR 2.575, 95\% CI 2.386-2.779), with $23.6 \%$ of cranial procedures leading to complications compared with $11.2 \%$ of spinal procedures. The most frequent complication in the neurosurgical procedures overall or in either spinal or cranial procedures alone was bleeding requiring a transfusion, which occurred in $4.5 \%$ of patients, and was more likely to occur in cranial than in spinal patients (RR 1.255, 95\% CI 1.136-1.385). The second most common complication was reoperation within 30 days of the initial operation, and this occurred in $4.3 \%$ of neurosurgical patients overall and was more likely in cranial than in spinal patients (RR 2.11, 


\section{Complications in neurosurgery}

TABLE 3: Frequency of complications in 38,058 neurosurgical cases*

\begin{tabular}{|c|c|c|c|c|}
\hline Parameter & $\begin{array}{c}\text { Cranial Op } \\
(\%)\end{array}$ & Spinal Op (\%) & $\begin{array}{l}\text { Cranial \& Spinal } \\
\text { Op Combined }(\%)\end{array}$ & $\begin{array}{l}\text { RR, Cranial vs Spinal } \\
\quad(95 \% \mathrm{Cl})\end{array}$ \\
\hline no. of cases & 10,041 & 28,017 & & \\
\hline \multicolumn{5}{|l|}{ complication } \\
\hline superficial incisional infection & $72(0.7)$ & $301(1.1)$ & $373(1.0)$ & $0.667(0.517-0.862) \dagger$ \\
\hline deep incisional infection & $45(0.4)$ & $166(0.6)$ & $211(0.6)$ & $0.756(0.545-1.051)$ \\
\hline organ/space infection & $105(1.0)$ & $91(0.3)$ & $196(0.5)$ & $3.220(2.434-4.259) \dagger$ \\
\hline wound dehiscence & $24(0.2)$ & $82(0.3)$ & $106(0.3)$ & $0.817(0.519-1.286)$ \\
\hline postop pneumonia & $367(3.6)$ & $212(0.8)$ & $579(1.5)$ & $4.830(4.085-5.711) \dagger$ \\
\hline unplanned reintubation & $300(3.0)$ & $182(0.6)$ & $482(1.3)$ & $4.599(3.831-5.522) \dagger$ \\
\hline pulmonary embolism & $137(1.4)$ & $107(0.4)$ & $244(0.6)$ & $3.573(2.777-4.596) \dagger$ \\
\hline $\begin{array}{l}\text { failure to wean from ventilator for }>48 \\
\text { hrs postop }\end{array}$ & $759(7.6)$ & $191(0.7)$ & $950(2.5)$ & $11.088(9.477-12.973) \dagger$ \\
\hline progressive renal insufficiency & $24(0.2)$ & $36(0.1)$ & $60(0.2)$ & $1.860(1.110-3.116) \dagger$ \\
\hline acute renal failure requiring dialysis & $21(0.2)$ & $20(0.1)$ & $41(0.1)$ & $2.930(1.589-5.403) \dagger$ \\
\hline urinary tract infection & $402(4.0)$ & $458(1.6)$ & $860(2.3)$ & $2.449(2.146-2.795) \dagger$ \\
\hline stroke/CVA & $219(2.2)$ & $42(0.1)$ & $261(0.7)$ & $14.549(10.466-20.225) \dagger$ \\
\hline coma (>24 hrs) & $113(1.1)$ & $5(0.0)$ & $118(0.3)$ & $63.060(25.756-154.395) \dagger$ \\
\hline peripheral nerve injury & $10(0.1)$ & $32(0.1)$ & $42(0.1)$ & $0.872(0.429-1.773)$ \\
\hline cardiac arrest requiring CPR & $65(0.6)$ & $49(0.2)$ & $114(0.3)$ & $3.701(2.556-5.359) \dagger$ \\
\hline myocardial infarction & $34(0.3)$ & $54(0.2)$ & $88(0.2)$ & $1.757(1.145-2.697) \dagger$ \\
\hline bleeding requiring transfusion & $540(5.4)$ & $1201(4.3)$ & $1741(4.6)$ & $1.255(1.136-1.385) \dagger$ \\
\hline graft/prosthesis/flap failure & $2(0.0)$ & $7(0.0)$ & $9(0.0)$ & $0.797(0.166-3.837)$ \\
\hline deep venous thrombosis requiring therapy & $259(2.6)$ & $225(0.8)$ & $484(1.3)$ & $3.212(2.690-3.834) \dagger$ \\
\hline sepsis & $363(3.6)$ & $251(0.9)$ & $614(1.6)$ & $4.035(3.441-4.732) \dagger$ \\
\hline septic shock & $128(1.3)$ & $80(0.3)$ & $208(0.5)$ & $4.464(3.380-5.898) \dagger$ \\
\hline reop w/in 30 days & $738(7.3)$ & 899 (3.2) & $1637(4.3)$ & $2.113(2.013-2.217) \dagger$ \\
\hline any complication & $2373(23.6)$ & 3134 (11.2) & 5507 (14.5) & 2.575 (2.386-2.779)† \\
\hline
\end{tabular}

95\% CI 2.01-2.22). The third most common complication was failure to wean from mechanical ventilation for more than 48 hours postoperatively, which occurred in $2.5 \%$ of neurosurgical patients. In fact, all complications except 5 were more likely in cranial procedures than in spinal ones. Of these 5, there was no significant difference in 4 and only 1 in which spinal procedures were significantly more likely to generate a complication: superficial surgical site infection (cranial RR 0.67, 95\% CI 0.52-0.86). The 5 nonsignificantly different complications were wound dehiscence, deep surgical site infection, peripheral nerve injury, and graft, prosthesis, or flap failure.

Using univariate analysis, we examined potential patient predictors that might be associated with the occurrence of a complication (Table 4). Many of these factors showed a significant association with complications. For example, African Americans were 1.51 times more likely to experience a complication than whites, and Asians/ Pacific Islanders were 1.58 times more likely. Complications were also more likely to occur in inpatients (RR 4.95, 95\% CI 4.28-5.73), patients with diabetes (RR $1.39,95 \%$ CI 1.31-1.48), emergent cases (RR 3.83, 95\%
CI 3.64-4.04), and frequent users of alcohol (RR 1.36, 95\% CI 1.20-1.54). Altogether, 34 characteristics were significantly associated with complications on univariate analysis.

Using the predictors identified with univariate analysis, we then constructed a model using binary logistic regression to control for confounding. This analysis identified 15 significant predictors (Table 5). African American race continued to be a significant risk factor for developing complications (RR 1.24, 95\% CI 1.10-1.41), whereas American Indian or Native Alaskan race continued to be protective (RR $0.53,95 \%$ CI $0.28-0.98$ ). However, Asian or Pacific Islander race was no longer a significant risk factor.

Many risk factors continued to be significantly associated with complications, such as diabetes, prior stroke, open wound, chronic steroid use, presurgical sepsis, and the need for a preoperative transfusion with $>4$ units of red blood cells (Table 5). Cranial procedures still produced more complications than spinal procedures (RR $1.41,95 \% 1.27-1.56)$, despite controlling for patient comorbidities. 
J. D. Rolston et al.

TABLE 4: Univariate predictors of complications among 5495 neurosurgical patients*

\begin{tabular}{|c|c|c|}
\hline Factor & No. of Complications (\%) & $\mathrm{RR}(95 \% \mathrm{Cl})$ \\
\hline \multicolumn{3}{|l|}{$\operatorname{sex}$} \\
\hline male & $2685(48.9)$ & $1 \dagger$ \\
\hline female & $2810(51.1)$ & $1.09(1.03-1.14)$ \\
\hline \multicolumn{3}{|l|}{ racef } \\
\hline white & $4083(74.1)$ & $1 \dagger$ \\
\hline black or African American & $596(10.8)$ & $1.51(1.39-1.62) \S$ \\
\hline Asian or Pacific Islander & $147(2.7)$ & $1.58(1.36-1.82) \S$ \\
\hline American Indian or native Alaskan & $21(0.4)$ & $0.70(0.47-1.05)$ \\
\hline \multicolumn{3}{|l|}{ spinal vs cranial } \\
\hline spinal & $3134(56.9)$ & $1 \dagger$ \\
\hline cranial & $2373(43.1)$ & $2.11(2.01-2.22) \S$ \\
\hline \multicolumn{3}{|l|}{ inpatient vs outpatient } \\
\hline outpatient & $179(3.3)$ & $1 \dagger$ \\
\hline inpatient & $5328(96.7)$ & $4.95(4.28-5.73) \S$ \\
\hline \multicolumn{3}{|l|}{ diabetes } \\
\hline no & $4470(81.2)$ & $1 \dagger$ \\
\hline yes & $1037(18.8)$ & $1.39(1.31-1.48) \S$ \\
\hline insulin-dependent & $439(8.0)$ & $1.68(1.54-1.83) \S$ \\
\hline not insulin-dependent & $598(10.9)$ & $1.24(1.15-1.34) \S$ \\
\hline \multicolumn{3}{|l|}{ tobacco use } \\
\hline no & $4265(77.4)$ & $1 \dagger$ \\
\hline yes & $1242(22.6)$ & $0.86(0.81-0.91) \S$ \\
\hline \multicolumn{3}{|l|}{ alcohol use } \\
\hline no & $3827(94.8)$ & $1 \dagger$ \\
\hline yes & $208(5.2)$ & $1.36(1.20-1.54) \S$ \\
\hline \multicolumn{3}{|l|}{ dyspneał } \\
\hline no & $4985(90.5)$ & $1 \dagger$ \\
\hline moderate exertion & $405(7.4)$ & $1.32(1.20-1.44) \S$ \\
\hline at rest & $116(2.1)$ & $2.92(2.53-3.36) \S$ \\
\hline \multicolumn{3}{|l|}{ ventilator dependent prior to op } \\
\hline no & $4977(90.4)$ & $1 \dagger$ \\
\hline yes & $530(9.6)$ & $6.06(5.79-6.34) \S$ \\
\hline \multicolumn{3}{|l|}{ severe COPD } \\
\hline no & $5146(93.4)$ & $1 \dagger$ \\
\hline yes & $361(6.6)$ & $1.65(1.50-1.81) \S$ \\
\hline \multicolumn{3}{|l|}{ current pneumoniał } \\
\hline no & $3988(99.0)$ & $1 \dagger$ \\
\hline yes & $41(1.0)$ & $4.10(3.37-4.99) \S$ \\
\hline \multicolumn{3}{|l|}{ congestive heart failuref } \\
\hline no & $5538(98.7)$ & $1 \dagger$ \\
\hline yes & $69(1.3)$ & $3.79(3.23-4.45) \S$ \\
\hline \multicolumn{3}{|l|}{ MI w/in 6 mosł } \\
\hline no & 3992 (99.1) & $1 \dagger$ \\
\hline yes & $37(0.9)$ & $3.31(2.62-4.18) \S$ \\
\hline \multicolumn{3}{|l|}{ angina in past moł } \\
\hline no & 3998 (99.3) & $1 \dagger$ \\
\hline yes & $30(0.7)$ & $1.44(1.05-1.98) \S$ \\
\hline
\end{tabular}

(continued) 


\section{Complications in neurosurgery}

TABLE 4: Univariate predictors of complications among 5495 neurosurgical patients* (continued)

\begin{tabular}{|c|c|c|}
\hline Factor & No. of Complications (\%) & $\mathrm{RR}(95 \% \mathrm{Cl})$ \\
\hline \multicolumn{3}{|c|}{ hypertensive patient on antihypertensive(s) } \\
\hline no & $2390(43.4)$ & $1 \dagger$ \\
\hline yes & $3117(56.6)$ & $1.48(1.40-1.55) \S$ \\
\hline \multicolumn{3}{|c|}{ prior angioplasty/amputation for PVD } \\
\hline no & $3963(98.4)$ & $1 \dagger$ \\
\hline yes & $66(1.6)$ & $1.73(1.40-2.14) \S$ \\
\hline \multicolumn{3}{|l|}{ renal failure on dialysis } \\
\hline no & $5428(98.6)$ & $1 \dagger$ \\
\hline yes & $79(1.4)$ & $3.49(2.98-4.09) \S$ \\
\hline \multicolumn{3}{|l|}{ altered mental status $\ddagger$} \\
\hline no & $3461(85.9)$ & $1 \dagger$ \\
\hline yes & $568(14.1)$ & $4.13(3.87-4.40) \S$ \\
\hline \multicolumn{3}{|l|}{ coma prior to opł } \\
\hline no & $3845(95.4)$ & $1 \dagger$ \\
\hline yes & $184(4.6)$ & $5.84(5.46-.25) \S$ \\
\hline \multicolumn{3}{|c|}{ hemiplegia/hemiparesis prior to op $\ddagger$} \\
\hline no & $3592(89.2)$ & $1 \dagger$ \\
\hline yes & $437(10.8)$ & $2.73(2.52-2.95) \S$ \\
\hline \multicolumn{3}{|c|}{ history of transient ischemic attack(s)ł } \\
\hline no & $3888(96.5)$ & $1 \dagger$ \\
\hline yes & $141(3.5)$ & $1.49(1.28-1.73) \S$ \\
\hline \multicolumn{3}{|c|}{ prior stroke w/ persistent neurological deficitł } \\
\hline no & $3504(87.0)$ & $1 \dagger$ \\
\hline yes & $525(13.0)$ & $3.50(3.26-3.75) \S$ \\
\hline \multicolumn{3}{|l|}{ prior stroke w/ no deficitł } \\
\hline no & $3875(96.2)$ & $1 \dagger$ \\
\hline yes & $154(3.8)$ & $2.04(1.78-2.34) \S$ \\
\hline \multicolumn{3}{|l|}{ CNS tumor } \\
\hline no & $3349(83.1)$ & $1 \dagger$ \\
\hline yes & $680(16.9)$ & $1.72(1.60-1.85) \S$ \\
\hline \multicolumn{3}{|c|}{ paraplegia/paraparesis prior to op $\ddagger$} \\
\hline no & $3731(92.6)$ & $1 \dagger$ \\
\hline yes & $298(7.4)$ & $1.90(1.72-2.11) \S$ \\
\hline \multicolumn{3}{|c|}{ quadriplegia/quadriparesis prior to op } \\
\hline no & $3944(97.9)$ & $1 \dagger$ \\
\hline yes & $85(2.1)$ & $2.51(2.11-2.98) \S$ \\
\hline \multicolumn{3}{|c|}{ disseminated/metastatic cancerł } \\
\hline no & $5048(92.3)$ & $1 \dagger$ \\
\hline yes & $423(7.7)$ & $2.00(1.83-2.17) \S$ \\
\hline \multicolumn{3}{|l|}{ open wound } \\
\hline no & $5279(95.9)$ & $1 \dagger$ \\
\hline yes & $228(4.1)$ & $2.56(2.30-2.85) \S$ \\
\hline \multicolumn{3}{|l|}{ chronic steroid use } \\
\hline no & $4994(90.7)$ & $1 \dagger$ \\
\hline yes & $513(9.3)$ & $1.58(1.46-1.71) \S$ \\
\hline \multicolumn{3}{|c|}{$>10 \%$ weight loss in last 6 mos } \\
\hline no & $5389(97.9)$ & $1 \dagger$ \\
\hline yes & $118(2.1)$ & $1.94(1.66-2.26) \S$ \\
\hline
\end{tabular}

(continued) 
TABLE 4: Univariate predictors of complications among 5495 neurosurgical patients* (continued)

\begin{tabular}{|c|c|c|}
\hline Factor & No. of Complications (\%) & $\mathrm{RR}(95 \% \mathrm{Cl})$ \\
\hline \multicolumn{3}{|l|}{ bleeding disorder } \\
\hline no & $5124(93.0)$ & $1 \dagger$ \\
\hline yes & $383(7.0)$ & $2.53(2.33-2.76) \S$ \\
\hline \multicolumn{3}{|c|}{ transfused $>4$ units RBCs prior to op } \\
\hline no & $5416(98.3)$ & $1 \dagger$ \\
\hline yes & $91(1.7)$ & $4.93(3.87-4.99) \S$ \\
\hline \multicolumn{3}{|l|}{ chemotherapy in past moł } \\
\hline no & 3941 (97.8) & $1 \dagger$ \\
\hline yes & $88(2.2)$ & $2.22(1.87-2.65) \S$ \\
\hline \multicolumn{3}{|c|}{ radiotherapy in past 90 days $\ddagger$} \\
\hline no & $3943(98.6)$ & $1 \dagger$ \\
\hline yes & $56(1.4)$ & $2.23(1.80-2.78) \S$ \\
\hline \multicolumn{3}{|l|}{ sepsis prior to op $\ddagger$} \\
\hline no & $4870(89.0)$ & $1 \dagger$ \\
\hline SIRS & $426(7.8)$ & $3.57(3.32-3.84) \S$ \\
\hline sepsis & $143(2.6)$ & $3.82(3.40-4.30) \S$ \\
\hline septic shock & $32(0.6)$ & $4.07(3.22-5.16) \S$ \\
\hline \multicolumn{3}{|l|}{ emergency case } \\
\hline no & $4437(80.6)$ & $1 \dagger$ \\
\hline yes & $1070(19.4)$ & $3.83(3.64-4.04) \S$ \\
\hline \multicolumn{3}{|l|}{ admission quarter } \\
\hline $1 \mathrm{st}$ & $1201(21.8)$ & $1 \dagger$ \\
\hline 2nd & $1127(20.5)$ & $1.00(0.93-1.08)$ \\
\hline $3 r d$ & $1507(27.4)$ & $1.06(0.99-1.13)$ \\
\hline 4th & $1672(30.4)$ & $1.08(1.00-1.15) \S$ \\
\hline
\end{tabular}

\section{Discussion}

Minimizing surgical complications has been a goal in neurosurgery ever since Harvey Cushing began rigorously documenting his own surgical errors more than a century ago. ${ }^{12}$ However, understanding and mitigating complications has taken on renewed importance in the era of "pay for performance" and governmental and insurance reimbursement metrics., ${ }^{5,6,15,20,22}$ Therefore, we sought to describe the current state of complications in neurological surgery, to provide data to help understand how new regulations should be applied, and, of course, to better target interventions to improve patient well-being.

Complications occurred in $14.3 \%$ of neurosurgical cases. The most frequent complications, in either cranial or spinal operations, were blood loss requiring transfusion and reoperation within 30 days, followed, somewhat surprisingly, by failure to wean from mechanical ventilation for $>48$ hours postoperatively, which occurred in $7.6 \%$ of cranial cases and $0.7 \%$ of spinal cases. This latter complication deserves note because, in our experience, it is seldom explicitly discussed with patients preoperative- ly, even though empirically it is one of the most frequent complications. The reasons for the high prevalence of this complication deserve more study.

Overall, complications were far more likely to occur in cranial procedures than in spinal ones $(23.6 \%$ cranial vs $11.2 \%$ spinal). Even when accounting for other patient risk factors, cranial procedures were still 1.41 times more likely to involve complications than spinal procedures (95\% CI 1.27-1.56). The source of this discrepancy is unclear from the data captured within the NSQIP data set. It should be stressed, however, that the NSQIP is only an incomplete reflection of patient characteristics. The nature of diseases bringing one to cranial neurosurgery (for example, glioma, hydrocephalus) are far different from those for spine surgery (for example, herniated intervertebral discs, spinal instability), and additional data on patient characteristics and risk factors not present in the NSQIP database could contribute to the observed difference in complication frequencies.

Many of the predictors of complications were expected and are traditionally considered as high-risk comorbidities from a pathophysiological standpoint (for ex- 


\section{Complications in neurosurgery}

TABLE 5: Significant multivariate predictors of complications

\begin{tabular}{|c|c|}
\hline Factor & OR $(95 \% \mathrm{Cl})$ \\
\hline \multicolumn{2}{|l|}{ sex } \\
\hline female & $1^{*}$ \\
\hline male & $0.90(0.83-0.97)$ \\
\hline \multicolumn{2}{|l|}{ race } \\
\hline white & $1^{*}$ \\
\hline black or African American & $1.24(1.10-1.41) \dagger$ \\
\hline Asian or Pacific Islander & $1.28(0.98-1.65) \dagger$ \\
\hline American Indian or native Alaskan & $0.53(0.28-0.98) \dagger$ \\
\hline \multicolumn{2}{|l|}{ spinal vs cranial } \\
\hline spinal & $1^{*}$ \\
\hline cranial & $1.41(1.27-1.56) \dagger$ \\
\hline \multicolumn{2}{|l|}{ inpatient vs outpatient } \\
\hline inpatient & $1^{*}$ \\
\hline outpatient & $0.31(0.26-0.37) \dagger$ \\
\hline \multicolumn{2}{|l|}{ diabetes } \\
\hline no & $1^{*}$ \\
\hline insulin-dependent & $1.30(1.11-1.53) \dagger$ \\
\hline not insulin-dependent & $1.08(0.95-1.23)$ \\
\hline \multicolumn{2}{|l|}{ alcohol use } \\
\hline no & $1^{*}$ \\
\hline yes & $1.25(1.04-1.52) \dagger$ \\
\hline \multicolumn{2}{|l|}{ dyspnea } \\
\hline no & $1^{*}$ \\
\hline moderate exertion & $1.05(0.71-1.56)$ \\
\hline at rest & $1.17(1.00-1.37) \dagger$ \\
\hline \multicolumn{2}{|l|}{ ventilator dependent prior to op } \\
\hline no & $1^{*}$ \\
\hline yes & $5.16(3.79-7.03) \dagger$ \\
\hline \multicolumn{2}{|l|}{ severe COPD } \\
\hline no & $1^{*}$ \\
\hline yes & $1.34(1.12-1.60) \dagger$ \\
\hline \multicolumn{2}{|l|}{ congestive heart failure } \\
\hline no & $1^{*}$ \\
\hline yes & $1.73(1.01-2.96) \dagger$ \\
\hline \multicolumn{2}{|c|}{ hypertensive patient on antihypertensive(s) } \\
\hline no & $1^{*}$ \\
\hline yes & $1.16(1.06-1.27) \dagger$ \\
\hline \multicolumn{2}{|l|}{ renal failure on dialysis } \\
\hline no & $1^{*}$ \\
\hline yes & $2.03(1.29-3.21) \dagger$ \\
\hline \multicolumn{2}{|l|}{ altered mental status } \\
\hline no & $1^{*}$ \\
\hline yes & $1.59(1.33-1.91) \dagger$ \\
\hline \multicolumn{2}{|c|}{ prior stroke w/ persistent neurological deficit } \\
\hline no & $1^{*}$ \\
\hline yes & $1.60(1.34-1.91) \dagger$ \\
\hline \multicolumn{2}{|l|}{ prior stroke w/ no deficit } \\
\hline no & $1^{*}$ \\
\hline yes & $1.81(1.44-2.28) \dagger$ \\
\hline
\end{tabular}

(continued)
TABLE 5: Significant multivariate predictors of complications (continued)

\begin{tabular}{|c|c|}
\hline Factor & OR $(95 \% \mathrm{Cl})$ \\
\hline \multicolumn{2}{|c|}{ paraplegia/paraparesis prior to op } \\
\hline no & $1^{*}$ \\
\hline yes & $1.92(1.63-2.26) \dagger$ \\
\hline \multicolumn{2}{|c|}{ quadriplegia/quadriparesis prior to op } \\
\hline no & $1^{*}$ \\
\hline yes & $2.63(1.93-3.59) \dagger$ \\
\hline \multicolumn{2}{|l|}{ open wound } \\
\hline no & $1^{*}$ \\
\hline yes & $1.60(1.25-2.04) \dagger$ \\
\hline \multicolumn{2}{|l|}{ chronic steroid use } \\
\hline no & $1^{*}$ \\
\hline yes & $1.28(1.10-1.48) \dagger$ \\
\hline \multicolumn{2}{|c|}{ preop transfusion $\mathrm{w} />4$ units RBCs } \\
\hline no & $1^{*}$ \\
\hline yes & $5.02(3.07-8.22) \dagger$ \\
\hline \multicolumn{2}{|l|}{ sepsis prior to op } \\
\hline no & $1^{*}$ \\
\hline SIRS & $2.07(1.69-2.54) \dagger$ \\
\hline sepsis & $2.37(1.70-3.31) \dagger$ \\
\hline septic shock & $0.82(0.41-1.65)$ \\
\hline \multicolumn{2}{|l|}{ brain or spine tumor } \\
\hline no & $1^{*}$ \\
\hline yes & $1.42(1.24-1.61) \dagger$ \\
\hline \multicolumn{2}{|l|}{ emergency case } \\
\hline no & $1^{*}$ \\
\hline yes & $2.27(1.96-2.64) \dagger$ \\
\hline
\end{tabular}

ample, presurgical sepsis, prior stroke, diabetes). Others were somewhat harder to explain. For example, African Americans are significantly more likely to experience complications than whites, with an RR of 1.24 (95\% CI 1.10-1.41). This racial/ethnic disparity in surgical complication rates has been described across multiple surgical disciplines; however, the exact source of this bias is unknown. $3,7,14,18,21$ Although many comorbid conditions were controlled for in our multivariate model, it is possible that some untracked characteristics in African Americans make them more vulnerable to complications (for example, disease type or severity) or that socioeconomic factors (for example, access to care) are leading to more complications. In either case, we must seek the cause of this disparity to improve the health of our patients.

Several limitations to our study are worth noting. The NSQIP database is a well-curated set of records in which trained analysts enter every complication using strict criteria. Nonetheless, it has several inherent limitations, especially as regards neurosurgical patients. First, the 30-day analysis of complications that is standardized across all sites from which data are gathered is a major strength of the NSQIP database; however, a number of complications 
unique to the neurosurgical population may not be accurately represented. For example, delayed hemorrhage from an incompletely treated vascular malformation would not be recorded, and there would be no distinction between a postoperative neurological deficit that is permanent versus one that resolves over time. Tracked complications are, by necessity, general and cover a wide range of categories: incision related (both infection and dehiscence), pulmonary (pneumonia, failure to wean from ventilator, and so forth), urinary tract infections, strokes, cardiac arrest or myocardial infarction, renal failure, bleeding, peripheral nerve injury, deep venous thrombosis or pulmonary embolism, sepsis, and reoperation. The NSQIP data set is used by many surgical disciplines, and complications unique to neurosurgery and related disciplines are irrelevant to the majority of the database's users (for example, CSF leaks, pseudomeningoceles). Similarly, counting complications by using broad measures may not reflect the nuances encountered in neurosurgical care. For example, rates of failure to wean from a ventilator within 48 hours probably included patients with planned postoperative mechanical ventilation to manage seizures or to control intracranial pressure. In addition, preoperative morbidity variables are similarly generalized and broad. The categories include race, sex, history of tobacco or alcohol use, history of bleeding disorders, transfusions, chemo- or radiotherapy, sepsis, diabetes, disseminated malignancy, and a host of common pulmonary, cardiac, and renal conditions. Variables more relevant to neurosurgery included an altered mental status, history of stroke or transient ischemic attack, paralysis (hemiplegia, paraplegia, quadriplegia), or a CNS tumor. However, the presence of a CNS tumor is a "yes/ no" field in the NSQIP database, which ignores distinctions between benign tumors such as meningiomas versus aggressive tumors such as glioblastomas. The database could have even greater value for the neurosurgical community if it included complications that are unique to, but represent a significant burden, to neurosurgical patients, such as postoperative seizures or meningitis. In the same regard, to facilitate more refined risk adjustment in the neurosurgical population, we must collect risk adjustment variables long suspected to be predictors of complications in neurosurgery such as history of prior craniotomies or cranial/spinal radiation or radiosurgery.

These issues highlight the need for neurosurgeryspecific databases, which will track relevant complications and comorbidities. Some exciting progress has been made on such databases, particularly with the development of the National Neurosurgery Quality and Outcomes Database $\left(\mathrm{N}^{2} \mathrm{QOD}\right)$, sponsored by the NeuroPoint Alliance and managed by Vanderbilt's Institute for Medicine and Public Health. ${ }^{1,2,13}$ Neurosurgery-specific databases will go a long way in addressing these concerns, and current efforts to prospectively collect neurosurgeryspecific data are important steps toward optimizing the care of patients.

\section{Conclusions}

Complications endanger patients and deplete scarce health care resources. To defend against complications, we attempted, first, to understand their prevalence and, second, to identify factors predictive of their occurrence. Analyzing data from more than 38,000 neurosurgical patients in the NSQIP database, we found that the most frequent complications were blood loss requiring transfusion and reoperation within 30 days, followed by failure to wean from mechanical ventilation postoperatively. We also found that complications were more frequent in cranial than in spinal procedures and more likely to occur in African Americans than in whites. The sources of these disparities remain to be determined, and further research with particular attention to complications unique to neurosurgery is clearly necessary. Once we define the most costly and harmful complications, we can begin to rationally direct our quality improvement efforts.

\section{Acknowledgments}

The ACS NSQIP and the hospitals participating in the ACS NSQIP are the sources of the data used herein; however, they have not verified and are not responsible for the statistical validity of our data analysis or derived conclusions.

We thank Rita Mistry, M.P.H., for her input on the manuscript and her expertise.

\section{Disclosure}

Dr. Parsa was supported in part by the Reza and Georgianna Khatib Endowed Chair in Skull Base Tumor Surgery. This work was supported in part by a Socioeconomic Fellowship from the Congress of Neurological Surgeons (J.R.). The authors report no conflict of interest concerning the materials or methods used in this study or the findings specified in this paper.

Author contributions to the study and manuscript preparation include the following. Conception and design: all authors. Acquisition of data: all authors. Analysis and interpretation of data: all authors. Drafting the article: all authors. Critically revising the article: all authors. Reviewed submitted version of manuscript: all authors. Approved the final version of the manuscript on behalf of all authors: Parsa. Study supervision: Parsa.

\section{References}

1. Asher AL, McCormick PC, Selden NR, Ghogawala Z, McGirt MJ: The National Neurosurgery Quality and Outcomes Database and NeuroPoint Alliance: rationale, development, and implementation. Neurosurg Focus 34(1):E2, 2013

2. Asher AL, McGirt MJ, Glassman SD, Groman R, Resnick DK, Mehrlich M, et al: Regulatory considerations for prospective patient care registries: lessons learned from the National Neurosurgery Quality and Outcomes Database. Neurosurg Focus 34(1):E5, 2013

3. Blum MA, Singh JA, Lee GC, Richardson D, Chen W, Ibrahim SA: Patient race and surgical outcomes after total knee arthroplasty: an analysis of a large regional database. Arthritis Care Res (Hoboken) 65:414-420, 2013

4. Dindo D, Demartines N, Clavien PA: Classification of surgical complications: a new proposal with evaluation in a cohort of 6336 patients and results of a survey. Ann Surg 240:205-213, 2004

5. Emanuel E, Tanden N, Altman S, Armstrong S, Berwick D, de Brantes F, et al: A systemic approach to containing health care spending. N Engl J Med 367:949-954, 2012

6. Glickman SW, Schulman KA, Peterson ED, Hocker MB, Cairns CB: Evidence-based perspectives on pay for performance and quality of patient care and outcomes in emergency medicine. Ann Emerg Med 51:622-631, 2008 


\section{Complications in neurosurgery}

7. Ibrahim SA, Stone RA, Han X, Cohen P, Fine MJ, Henderson WG, et al: Racial/ethnic differences in surgical outcomes in veterans following knee or hip arthroplasty. Arthritis Rheum 52:3143-3151, 2005

8. Ingraham AM, Richards KE, Hall BL, Ko CY: Quality improvement in surgery: the American College of Surgeons National Surgical Quality Improvement Program approach. Adv Surg 44:251-267, 2010

9. Kaafarani HM, Borzecki AM, Itani KM, Loveland S, Mull HJ, Hickson K, et al: Validity of selected patient safety indicators: opportunities and concerns. J Am Coll Surg 212:924-934, 2011

10. Khuri SF, Henderson WG, Daley J, Jonasson O, Jones RS, Campbell DA Jr, et al: Successful implementation of the Department of Veterans Affairs' National Surgical Quality Improvement Program in the private sector: the Patient Safety in Surgery Study. Ann Surg 248:329-336, 2008

11. Koch CG, Li L, Hixson E, Tang A, Phillips S, Henderson JM: What are the real rates of postoperative complications: elucidating inconsistencies between administrative and clinical data sources. J Am Coll Surg 214:798-805, 2012

12. Latimer K, Pendleton C, Olivi A, Cohen-Gadol AA, Brem H, Quiñones-Hinojosa A: Harvey Cushing's open and thorough documentation of surgical mishaps at the dawn of neurologic surgery. Arch Surg 146:226-232, 2011

13. McGirt MJ, Speroff T, Dittus RS, Harrell FE Jr, Asher AL: The National Neurosurgery Quality and Outcomes Database (N2QOD): general overview and pilot-year project description. Neurosurg Focus 34(1):E6, 2013

14. Mukherjee D, Patil CG, Todnem N, Ugiliweneza B, Nuño M, Kinsman M, et al: Racial disparities in Medicaid patients after brain tumor surgery. J Clin Neurosci 20:57-61, 2013

15. Nelson AR: Pay-for-performance programs: ethical questions and unintended consequences. J Fam Pract 56 (10 Suppl A): 16A-18A, 2007

16. Porter ME: What is value in health care? N Engl J Med 363: 2477-2481, 2010

17. Romano PS, Geppert JJ, Davies S, Miller MR, Elixhauser A, McDonald KM: A national profile of patient safety in U.S. hospitals. Health Aff (Millwood) 22:154-166, 2003

18. Scarborough JE, Bennett KM, Pappas TN: Racial disparities in outcomes after appendectomy for acute appendicitis. Am J Surg 204:11-17, 2012

19. Shiloach M, Frencher SK Jr, Steeger JE, Rowell KS, Bartzokis $\mathrm{K}$, Tomeh MG, et al: Toward robust information: data quality and inter-rater reliability in the American College of Surgeons National Surgical Quality Improvement Program. J Am Coll Surg 210:6-16, 2010

20. Song Z, Cutler DM, Chernew ME: Potential consequences of reforming Medicare into a competitive bidding system. JAMA 308:459-460, 2012

21. Vogel TR: Update and review of racial disparities in sepsis. Surg Infect (Larchmt) 13:203-208, 2012

22. Woolhandler S, Ariely D, Himmelstein DU: Why pay for performance may be incompatible with quality improvement. BMJ 345:e5015, 2012

Manuscript submitted December 16, 2012.

Accepted October 10, 2013.

Please include this information when citing this paper: published online November 22, 2013; DOI: 10.3171/2013.10.JNS122419.

Address correspondence to: Andrew T. Parsa, M.D., Ph.D., Department of Neurological Surgery, University of California, San Francisco, 505 Parnassus Ave., M779, San Francisco, CA 941430112. email: aparsa@nmff.org. 